\title{
Phenotypic and genotypic detection of metallo- $\beta$-lactamases in $A$. baumanii isolates obtained from clinical samples in Shahrekord, southwest Iran
}

\author{
Mansoor Khaledi, Milad Shahini Shams Abadi, Majid Validi, Behnam Zamanzad, Rezvan Vafapour \\ and Abolfazl Gholipour ${ }^{*}$
}

\begin{abstract}
Objective: Acinetobacter baumanii is a pathogenic bacterium that is the cause of many nosocomial infections. This study aimed to determine metallo- $\beta$-lactamases (MBL) produced by the A. baumanii isolates obtained from clinical samples in Shahrekord, southwest Iran.

Results: A total of 100 A. baumanii were isolated from 250 clinical samples between June 2013 and June 2014. Then, the isolates were identified by biochemical tests, and MBL screening was conducted by the phenotypic tests modified Hodge, EDTA-disk synergy (EDS), combined disk (CD) and AmpC disc after antibiotic sensitivity test. Using PCR technique the bla genes were detected. Eighty-five (85\%) isolates were resistant to meropenem and imipenem. Phenotypic tests showed that out of the 100 isolates, 46, 59, 50, 65 and 65 isolates were positive: AmpC disk, CD, EDS, Modified Hodge and E-test MBL respectively. Gene detection by PCR showed that 23 isolates carried the VIM-1 gene and only three isolates carried the IMP-1 gene. The prevalence of metallo- $\beta$-lactamases isolates containing A. baumanii is increasing. Furthermore, the coexistence of various carbapenemases is dominantly act as a major problem. Continuous monitoring of the infections related to these bacteria should be considered to plan an alternative and new therapeutic strategies.
\end{abstract}

Keywords: Acinetobacter baumanii, Metallo-beta-lactamase, Carbapenems, Iran

\section{Introduction}

Acinetobacter baumanii is recognized for human as a pathogenic bacterium that has the potential to acquire antibiotic resistance and significant inherent resistance in latest years. A. baumanii can survive in distinct circumstances of the environment. This bacterium is the most common pathogen that is responsible for nosocomial infections, including hospital-acquired pneumonia and urinary tract, central nervous system, skin, smooth tissue, bloodstream, bone, and surgical site infections [1]. A. baumanii's antibiotic resistance is one of the

*Correspondence: gholipour_abolfazl@yahoo.com Department of Microbiology and Immunology, Cellular and Molecular Research Center, Basic Health Sciences Institute, Shahrekord University of Medical Sciences, Shahrekord, Islamic Republic of Iran reasons why such diseases spread because such bacteria can intrinsically transfer resistance factors to each other [2]. Therefore, the treatment of A. baumanii infections have recently become challenging due to acquisition of resistance to numerous antibiotics by intrinsic and acquired mechanisms [3, 4]. Carbapenems are a class of antibiotics that are effective on gram-negative and gram-positive bacteria [3, 4]. These pathogens are resistant to penicillinases and cephalosporins [5]. The main antibiotic resistance mechanism in these classes is carbapenemase synthesis $[6,7]$. These carbapenems can be hydrolyzed by a class of $\beta$-lactamases categorized as B subclass by Ambler's classification and known as metallo$\beta$-lactamases (MBL). MBLs lead to numerous antibiotics resistance including penicillin, cephalosporins and 
carbapenems [5]. Decreased permeability of the outer membrane and increased efflux pump are the other mechanisms of carbapenem resistance $[8,9]$. There are a number of MBLs genes such as imipenemase (IMP), Verona integron-encoded metallo-beta-lactamases (VIM), Sno Paolo metallo (SPM), New-Delhi metallo- $\beta$ lactamase (NDM), German imipenemase (GIM), Kyorin University Hospital imipenemase (KHM), and Australian imipenemase (AIM) [10, 11]. IMP, VIM and SPM are the most important genes of MBLs that have been detected in $A$. baumanii [12]. It has been suggested that different phenotypic tests identify MBLs based on metal-chelating ability such as EDTA inhibiting MBL activity [13]. The phenotypic experiments were used in the present research to modify Hodge, double disk synergy (DDS), and $\mathrm{AmpC}$ disk tests. This research was carried out using phenotypic and genotypic methods to identify MBLs generated baumanii isolates. All the isolates obtained from clinical samples at teaching hospitals in Shahrekord, southwest Iran.

\section{Main text}

Materials and methods

Patients and sampling

Between June 2013 and May 2014, a total of 250 clinical samples including trachea, blood, urine, wound culture, cerebrospinal fluid, and pleural effusion was collected from the inpatients in different wards of Kashani and Hajar Hospitals of Shahrekord. This project was approved by Shahrekord University of Medical Sciences
Ethics Committee, and all informed participants complemented a written consent.

\section{Isolation and detection of $A$. baumanii isolates}

A. baumanii strains were isolated and identified by common chemical tests and techniques [14].

\section{Antimicrobial susceptibility test}

Antimicrobial susceptibility test was conducted by KirbyBauer protocol (disk diffusion) using $10 \mu \mathrm{g}$ imipenem and meropenem discs, E-test strips of these antibiotics, and MBL strip. Zone inhibition diameters were measured, and the results were interpreted with reference to the Clinical \& Laboratory Standards Institute (CLSI) (CLSI 2013). Pseudomonas aeruginosa (ATCC ${ }^{\circledR} 2785$ ) was used as qualitative control.

\section{Modified Hodge test}

A Modified Hodge test was conducted to screen for carbapenemase $[15,16]$. After suspension culture of Escherichia coli (ATCC 25922), a carbapenem-susceptible organism, for $24 \mathrm{~h}$ to reach a bacterial turbidity of 0.5 McFarland standard (NCCLS 2000). The bacteria were cultured in the Mueller-Hinton agar using a swab, and then the $10 \mu \mathrm{g}$ meropenem disk was placed in the center of the plate. After a 24-h culture, the bacteria did not grow around the disc. After a $24-\mathrm{h}$ incubation at $37^{\circ} \mathrm{C}$, the existence of inhibition zone was considered positive control (Fig. 1).

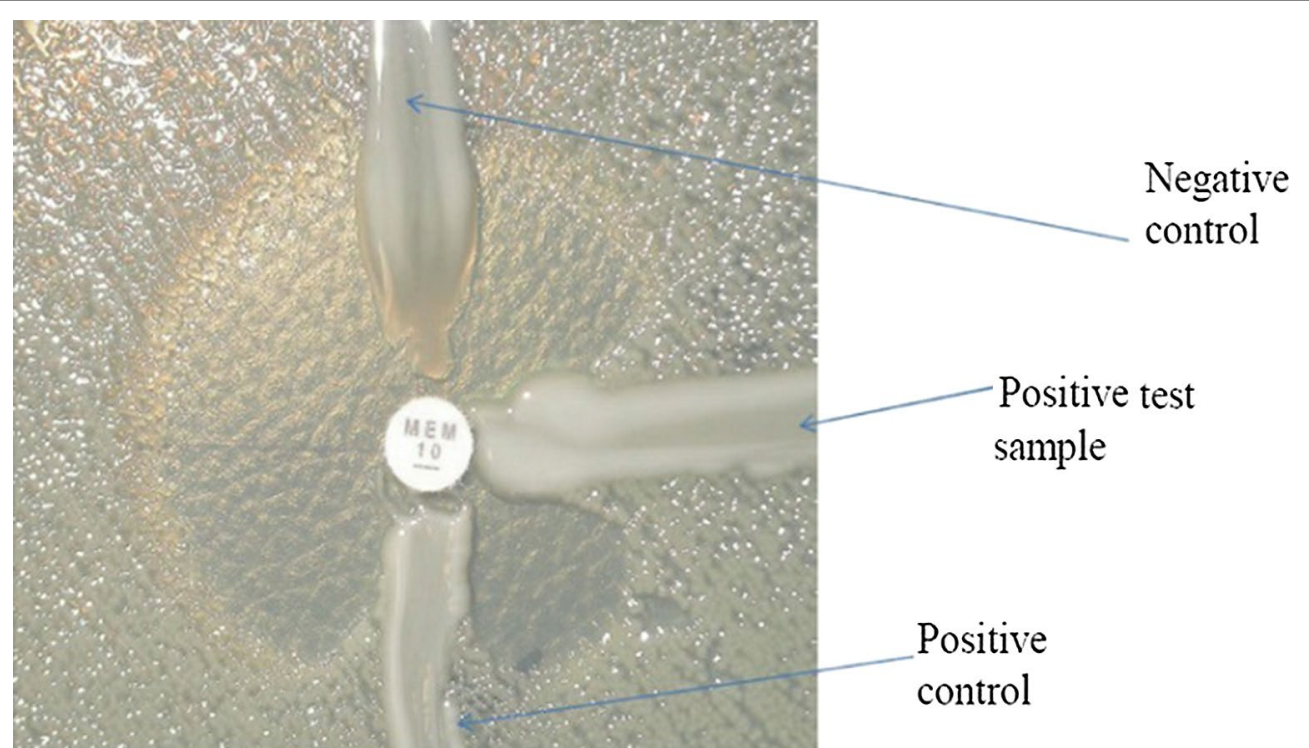

Fig. 1 Modified Hodge test. Positive strain shows a'cloverleaf shaped'zone of inhibition due to carbapenemase production, while the negative strain shows an undistorted zone of inhibition 


\section{EDS and CD tests}

EDS test was performed on meropenem- and imipenemresistant isolates using meropenem and ceftazidime, and CD test to detect MBLs $[16,17]$. Practically, a bacterial suspension of the $0.5 \mathrm{McFarland}$ standard was cultured on the Mueller-Hinton agar, and then two $10 \mu \mathrm{g}$ carbapenem (IMI and MEM) discs as well as a $30 \mu \mathrm{g}$ ceftazidime disc were added to the Mueller-Hinton agar. Subsequently, the MBL stop solution $(10 \mu \mathrm{l})$ was added to the culture medium. After a $24-\mathrm{h}$ incubation at $35{ }^{\circ} \mathrm{C}$, the inhibition zone diameter was measured and compared with other discs. For the EDS test, a bacterial suspension of the $0.5 \mathrm{McF}$ arland standard was prepared from the isolates, and the samples were cultured in Mueller-Hinton agar. A $10 \mu \mathrm{g}$ imeropenem disc was placed in the agar medium. A blank disc was kept in the inner surface of the Mueller-Hinton agar and $10 \mu \mathrm{l}$ of $0.5 \mathrm{M}$ EDTA was added. Likewise, the EDTA disc was transferred to the agar surface and kept at $10 \mathrm{~mm}$ distance from the meropenem or ceftazidime disc. After a 24-h incubation at $37{ }^{\circ} \mathrm{C}$, the presence of inhibition zone between the two discs was considered to represent MBL production.

\section{AmpC disk test}

AmpC disk test was conducted to detect MBL in imoperem-resistant strains [17-19].

\section{DNA extraction}

The DNAs of the bacterial isolates were extracted using a DNA extraction kit (Bioneer, Korea, Cat. No. K-3032-2-).

\section{Detection of bla (IMP) and bla (VIM) using PCR}

PCR was performed to screen for the VIM and IMP genes. Table 1 shows the used primers. PCR consisted of forward and reverse primers, DNA pattern, and Master Mix. The PCR product was analyzed by polyacrylamide gel.

\section{Data analysis}

Data including questionnaire data and the results of phenotypic and genotypic tests were analyzed by Chi square and Fisher's exact test in the SPSS version 22.

\section{Results}

Eighty-five (85\%) samples were resistant to meropenem and imipenem. 43 number of patients (43\%) were female. The mean age of the participants was 47.2 years (range; 1 dy- 88 years). The minor and major prevalence of isolated bacteria were allocated to the tracheal and abscess samples respectively.

The tracheal samples carried the most bacteria containing the MBLs. Fisher's exact test showed that the associations between the samples and the presence of bla VIM-1 $(p=0.28)$ and bla IMP-1 $(p=0.88)$ were not statistically significant.

\section{Resistance to meropenem and imipenem}

After incubation of the plates containing the meropenem and imipenem discs and E-test strips, the diameters of the inhibition zones were measured and interpreted with reference to the CLSI. For the E-test strips, minimum inhibitory concentration (MIC) was observed in the areas where inhibition zone diameters was increased. E-test with (MIC > 32) indicated that 85 samples of the $100 \mathrm{~A}$. baumanii isolates were resistant to meropenem and imipenem (carbapenem). Based on disk diffusion assay, 81 samples were resistant and four samples relatively resistant (semi-susceptible).

\section{The results of phenotypic tests for detecting MBLs} 46 (46\%) cases out of 100 strains were AmpC beta-lactamase producers, and 59 strains positive by CD test, representing MBL production. In addition, 50 samples were found to be positive in E-test (EDTA disk synergy test). Sixty-five samples were found as carbapenemase producing by the modified Hodge test and 65 as MBL producing by E-test.

\section{The results of genotypic tests}

After the electrophoresis of PCR products, the bands were carefully examined. The presence of the OXA51 gene (the specific internal gene of $A$. baumanii) was investigated to confirm the phenotypic tests for $A$. baumanii detection.

Table 1 PCR primers used in this study

\begin{tabular}{lll}
\hline Primers & Primer sequence & Product size \\
\hline VIM-1 F & TGGTTGTATACGTCCCGTCA:F & 206 bp \\
VIM-1 R & TGTGTGCTGGAGCAAGTCTA R: & bp 179 \\
IMP-1F & TAACGGGTGGGGCGTTGTTCCT:F & This study \\
IMP-1R & CGCCCGTGCTGTCGCTATGAAA R: & bp 353 \\
OXA-51 F & TAATGCTTTGATCGGCCTTG:F & \\
OXA-51 R & TGGATTGCACTTCATCTTGG R: & Hu et al. [26] \\
\hline
\end{tabular}


All samples carried the OXA-51 gene. The presence of this gene confirmed the results of biochemical tests for A. baumanii detection. The PCR results also showed that the $A$. baumanii isolates from our patients carried the VIM-1 and IMP-1 MBL genes. Twenty-three samples carried the VIM-1 gene and only three samples had the IMP-1 gene (Table 2).

\section{The association between meropenem resistance and the presence of bla IMP-1 and bla VIM-1 genes in Acinetobacter baumanii strains}

Chi square test $(\alpha=0.5)$ showed a significant association between meropenem resistance and the presence of bla VIM-1 gene in A. baumanii strains $(p=0.22)$; however, there was not relation between meropenem resistance and the presence of bla IMP-1 $(p=0.46)$.

\section{Discussion}

A. baumanii, a bacterium with multidrug resistance, is considered a highly important pathogen that can endanger human health. This organism is the cause of numerous infections in human, and appearances mainly in the people with immunodeficiency or underlying disease. The infections due to A. baumanii is treated with broad-spectrum antibiotics [9]. However, the antibiotic resistance of this microorganism has potentially been increased, like carbapenem resistance is spreading across the world [20]. Carbapenems including imipenem and meropenem are drugs of choice for A. baumanii infection. Recently, the emergence of MBLs (carbapenemase) belonging to the classes $\mathrm{D}$ and $\mathrm{B}$ of beta-lactamases has led to the hydrolysis of these drugs, and therefore, $A$. baumanii gain resistance to them [21, 22]. It is highly useful to early detection of these genes in carbapenem resistant bacteria to control and prevent the spread of these bacteria in hospitals. These data can also help the physicians to make appropriate prognosis and prescribe suitable antibiotic regimens [5]. In this study, we investigated the prevalence of the most important MBL genes that have been identified in A. baumanii strains.

We observed that $85(85 \%)$ isolates were resistant to meropenem and imipenem, and the prevalence of the IMP-1 and VIM-1 genes were derived 3\% and 23\% respectively.

Table 2 The VIM-1 and IMP-1 genes-producing isolates among meropenem- and imipenem-resistant Acinetobacter baumanii strains

\begin{tabular}{lllll}
\hline VIM-1 & & IMP-1 & & Total \\
\hline Positive & Negative & Positive & Negative & 100 \\
$23(23 \%)$ & $77(77 \%)$ & $3(3 \%)$ & $97(97 \%)$ & \\
\hline
\end{tabular}

Nouri et al. [23] studied the prevalence of MBLs in A. baumanii in Tehran with the most strains isolated from tracheal samples that is similar to our study. So, sterilization of respiratory tubes is one of the most important ways to prevent development of this infection in these tubes. In our study, the phenotypic tests AmpC disk, CD, EDS, modified Hodge, and E-Test MBL showed that 46, $59,50,65$, and 65 isolates in order were MBL-producing, and only 23 isolates carried the VIM- 1 gene and three isolates had the IMP-1 gene. This inconsistency in the findings can be attributed to the presence of other MBL genes or other mechanisms such as defects of porins or decreased expression of outer membrane proteins [24].

However, results of phenotypic and genotypic tests in several investigations have also consistence with our result. For example, Shoja reported that $55 \%$ of the isolates were positive by DDS test but did not find any MBL producing gene, which is consistent with the studies of Manageiro [25] and $\mathrm{Hu}$ [26].

Peymani et al. observed that among 63 carbapenemresistant $A$. baumanii strains, 31 (49\%) strains contained MBL which 19 (61\%) carried bla VIM-1 and the rest had bla IMP-1 (31\%). These statistics are consistent with our study with respect to the prevalence of the VIM-1 gene but inconsistent with respect to the prevalence of IMP-1 that was higher in the study of Peymani et al. [24]. The study of Kouyama et al. [27] indicated that out of the 598 A. baumanii strains isolated from the patients in the hospitals in different cities of Japan, $4.5 \%$ were resistant to imipenem and meropenem, which is a lower prevalence rate compared to different regions. This can be attributed to the genetic and climatic conditions in that region of the world. A. baumanii resistance rate to imipenem and meropenem is heterogeneous in East Asia. It has been reported 9.26\% in Korea [28], 49\% in Taiwan [29], $50-52.4 \%$ in China [30], and 4.5\% in Japan, possibly representing the effects of geographical differences.

Turner et al. [31] conducted a large study in Europe that showed imipenem resistance rate of $A$. baumanii isolates at the hospital level was $22-26 \%$. Sung et al. [32] reported that out of the 31 carbapenem-resistant $A$. baumanii strains, 15 (48.4\%) isolates carried the bla IMP-1 gene, which is comparatively higher than our finding. In regard to these statistics, the spread and prevalence of both genes are increasing. On the other hand, the lower prevalence of bla IMP-1 in the region studied in the current study and also the high prevalence and importance of bla VIM-1 should be paid special attention.

The coexistence of different carbapenemases is considered a serious health issue that have remarkable effects on alternative and newer therapeutic strategies, stricter measures of infection control, and new design for continuous monitoring. The continuous monitoring of the 
prevalence of carbapenem resistance and associated mechanisms in A. baumanii strains can help to develop appropriate therapeutic strategies against nosocomial infections.

\section{Limitations}

The lack of investigation on others resistance mechanisms in A. baumanii isolates can be mentioned as one of the main limitations of the present study.

\begin{abstract}
Abbreviations
MBL: metallo- $\beta$-lactamases; IMP: the genes imipenemase; VIM: Verona integron-encoded metallo-beta-lactamases; SPM: Sno Paolo metallo; NDM: New-Delhi metallo- $\beta$-lactamase; GIM: German imipenemase; KHM: Kyorin University Hospital imipenemase; AIM: Australian imipenemase.
\end{abstract}

\section{Acknowledgements}

Not applicable.

\section{Authors' contributions}

MK, MSSA, MV, AG: design of study. MK, BZ, RV, AG: acquisition of data. MK, MSSA, AG: evaluation of data, preparation of the manuscript. MV, BZ, RV, AG: assessment of data. All authors read and approved the final manuscript.

\section{Funding}

This research was supported by the budget of research projects of the Shahrekord University of medical sciences (approval code: 1392-01-74-1917). Funding body were used to purchase equipment and tools.

\section{Availability of data and materials}

All relevant data are included in the manuscript.

\section{Ethics approval and consent to participate}

This study was approved by the Ethics Committee of Shahrekord University of Medical Sciences. The informed consent was obtained from all the participants, and informed consent obtained was written.

\section{Consent to publish}

Not applicable.

\section{Competing interests}

The authors declare that they have no competing interests.

Received: 12 July 2019 Accepted: 11 September 2019

Published online: 18 September 2019

\section{References}

1. Peleg AY, Seifert H, Paterson DL. Acinetobacter baumannii: emergence of a successful pathogen. Clin Microbiol Res. 2008;21:538-82.

2. Dey D, Debnath S, Hazra S, Ghosh S, Ray R, Hazra B. Pomegranate pericarp extract enhances the antibacterial activity of ciprofloxacin against extended-spectrum beta-lactamase (ESBL) and metallo-betalactamase (MBL) producing Gram-negative bacilli. Food Chem Toxicol. 2012;50:4302-9.

3. van Looveren M, Goossens H. ARPAC Steering Group: antibacterial resistance of Acinetobacter spp. in Europe. Clin Microbiol Infect. 2004;10:684-704.

4. Navon-Venezia S, Ben-Ami R, Carmeli Y. Update on Pseudomonas aeruginosa and Acinetobacter baumannii infections in the healthcare setting. Curr Opin Infect Dis. 2005;18:306-13.

5. Nordmann P, Poirel L. Emerging carbapenemases in gram negative aerobes. Clin Microbiol Infect. 2002;8:321-31.

6. Soroush S, Haghi-Ashtiani MT, Taheri-Kalani M, et al. Antimicrobial resistance of nosocomial strain of Acinetobacter baumannii in children's medical center of Tehran: a 6-year prospective study. Acta Med Iran. 2010;48:178-84.

7. Asadollahi P, Akbari M, Soroush S, et al. Antimicrobial resistance patterns and their encoding genes among Acinetobacter baumannii strains isolated from burned patients. Burns. 2012;38:1198-203.

8. Amudhan SM, Sekar U, Arunagiri K, Sekar B. OXA Beta-lactamase-mediated carbapenem resistance in Acinetobacter baumannii. Indian J Med Microbiol. 2011;29:269-74.

9. Lin MF, Kuo HY, Yesh HW, Yang CM, Sug CH, Tu C. Emergence and dissemination of blaOXA-23 carrying imipenem-resistant Acinetobacter spp in a regional hospital in Taiwan. J Microbiol Immunol Infect. 2011;44:39-44.

10. Cornaglia G, Giamarellou H, Rossolini G. Metallo-betalactamases: a last frontier for beta-lactams? Lancet Infect Dis. 2011;11:381-93.

11. Fallah F, Hakemi VM, Goudarzi H, Hashemi A, Taherpour A, Bigdeli SK, et al. Identification of extended-spectrumbetalactamases(ESBLs), metallo-beta-lactamases (MBLs), Amp-C and KPC $B$ lactamases among Klebsiella pneumoniae isolated from adults and pediatric patients in Iran. African J Microbiol Res. 2013;7:3254-61.

12. Maragakis LL, Perl T. Acinetobacter baumannii: epidemiology, antimicrobial resistance, and treatment options. Clin Infect Dis. 2008;46:1254-63.

13. Saderi H, Karimi Z, Owlia P, Bahar A, Akhavi RSM. Phenotypic detection of metallobeta-lactamase-producing Pseudomonas aeruginosa strain isolated from burned patients. Iran J Pathol. 2008;3:20-4.

14. Shahcheraghi F, Abedin M. Molecular recognition bla IMP, bla SPM, bla VIM in Acinetobacter strains resistant to imipenem isolated from clinical specimens. Jlnfect Dis Trop Med. 2012;17:29-33.

15. Lee K, Chong Y, Shin HB, Kim YA, Yong D, Yum JH. Modified Hodge and EDTA-disk synergy tests to screen metollo-B-lactamase-producing strains of Pseudomonas and Acinetobacter species. Clin Microbiol Infect. 2001;7:88-102.

16. Eun-Jee O, Seungok L, Yeon-Joon P, Jung JP, Kanggyun P, Sang-II K, et al. Prevalence of metallo-h-lactamase among Pseudomonas aeruginosa and Acinetobacter baumannii in a Korean University hospital and comparison of screening methods for detecting metallo-h-lactamase. J Microbiol Methods. 2003;57:411-8.

17. Singhal S, Mathur T, Khan S, Upadhyay DJ, Chugh S, Gaind R, Rattan A. Evaluation of methods for AmpC $\beta$-lactamase in gram negative clinical isolates from tertiary care hospitals. Indian J Med Microbiol. 2005;23:120-4.

18. Dheepa M, Boppe A. Phenotypic methods for the detection of various betalactamases in carbapenem resistant isolates of Acinetobacter baumanii at a tertiary care hospital in South India. J Clin Diagn Res. 2012;6:970-3.

19. Walsh TR, Bolmstrom A, Qwarnstrom A, Gales A. Evaluation of a new Etest for detecting metallo-â-lactamases in routine clinical testing. J Clin Microbiol. 2002;40:2755-9.

20. Jeong SH, Bae IK, Park KO, An YJ, Sohn SG, Jang SJ, et al. Outbreaks of imipenem-resistant Acinetobacter baumannii producing carbapenemases in Korea. J Microbiol. 2006:44:423-33.

21. Versalovic J, Koeuth T, Lupski JR. Distribution of repetitive DNA sequences in eubacteria and application to fingerprinting of bacterial genomes. Nucleic Acids Res. 1991;19:6823-31.

22. Poirel L, Weldhagen GF, Naas T, De Champs C, Dove MG, Nordman P. GES-2, a class A beta-lactamase from Pseudomonas aeruginosa with increased hydrolysis of imipenem. Antimicrob Agents Chemother. 2001;45:2598-603.

23. Noori M, Karimi A, Fallah F, Hashemi A, Alimehr S, Goudarzi H, Aghamohammad S. High prevalence of metallo-beta-lactamase producing Acinetobacter baumannii isolated from two hospitals of Tehran, Iran. Arch Pediatr Infect Dis. 2014. https://doi.org/10.5812/pedinfect.15439.

24. Peymani A, Nahaei MR, Farajnia S, Hasani A, Mirsalehian A, Sohrabi N, Abbasi L. High prevalence of metallo-b-lactamase-producing Acinetobacter baumannii in a teaching hospital in Tabriz, Iran. Jpn J Infect Dis. 2011;64:69-71.

25. Manageiro V, Jones-Dias D, Ferreira E, Louro D, Canica M. Genetic diversity and clonal evolution of carbapenem-resistant Acinetobacter baumannii isolates from Portugal and the dissemination of ST118. Int J Antimicrob Agents. 2012;40:398-403.

26. Hu Q, Hu Z, Li J, Tian B, Xu H. Detection of OXA-type carbapenemases and integrons among carbapenem-resistant Acinetobacter baumannii in a teaching hospital in China. J Basic Microbiol. 2011;51:467-72. 
27. Kouyama Y, Harada S, Ishii Y, Saga T, Yoshizumi A, Tateda K, Yamaguchi K. Molecular characterization of carbapenem-non-susceptible Acinetobacter spp. in Japan: predominance of multidrug-resistant Acinetobacter baumannii clonal complex 92 and IMP-type metallo- $\beta$-lactamase-producing non-baumannii Acinetobacter species. J Infect Chem. 2012;1(18):522-8.

28. Jeon BC, Jeong SH, Bae IK, Kwon SB, Lee K, Young D, et al. Investigation of a nosocomial outbreak of imipenem-resistant Acinetobacter baumannii producing the OXA-23 b-lactamase in Korea. J Clin Microbiol. 2005:43:2241-5

29. Lin YC, Sheng WH, Chen YC, Chang SC, Hsia KC, Li SY. Differences in carbapenem resistance genes among Acinetobacter baumannii, Acinetobacter genospecies 3 and Acinetobacter genospecies 13TU in Taiwan. Int J Antimicrob Agents. 2010;35:439-43.

30. Xiao YH, Giske CG, Wei ZQ, Shen P, Heddini A, Li L. Epidemiology and characteristics of antimicrobial resistance in China. Drug Resist Update. 2011;14:236-50.
31. Turner P. activity of meropenem and other broad-spectrum agents against nosocomial isolates. Diagn Microbiol Infect Dis. 2009;63:217-22.

32. Sung JY, Kwon KC, Park JW, Kim YS, Kim JM, Shin KS, Kim JW, Ko CS, Shin SY, Song JH, Koo SH. Dissemination of IMP-1 and OXA type $\beta$-lactamase in carbapenem-resistant Acinetobacter baumannii. Korean J Lab Med. 2008;28:16-23

\section{Publisher's Note}

Springer Nature remains neutral with regard to jurisdictional claims in published maps and institutional affiliations.
Ready to submit your research? Choose BMC and benefit from:

- fast, convenient online submission

- thorough peer review by experienced researchers in your field

- rapid publication on acceptance

- support for research data, including large and complex data types

- gold Open Access which fosters wider collaboration and increased citations

- maximum visibility for your research: over $100 \mathrm{M}$ website views per year

At BMC, research is always in progress.

Learn more biomedcentral.com/submissions 